\title{
Anion-Triggered Substituent Dependent Conformational Switching of Salicylanilides. New Hints for Understanding Inhibitory Mechanism of Salicylanilides
}

\author{
Lin Guo, Qiang-Li Wang, Qian-Qian Jiang, Qiu-Ju Jiang and Yun-Bao Jiang* \\ Department of Chemistry, College of Chemistry and Chemical Engineering, and The MOE Key \\ Laboratory of Analytical Sciences, Xiamen University, Xiamen 361005, China \\ ybjiang@xmu.edu.cn
}

Supporting Information

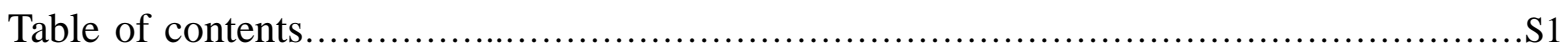

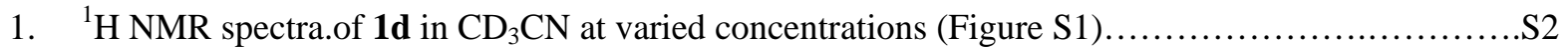

2. Chemical shift of $\mathrm{NH}$ proton of $\mathbf{1 d}$ in $\mathrm{CD}_{3} \mathrm{CN}$ as a function of $\mathbf{1 d}$ concentration (Figure S2) ......S2

3. Fluorescence spectra of salicylanilide 1d in the presence of triethylamine in $\mathrm{CH}_{3} \mathrm{CN}$ (Figure S3).

4. Absorption and fluorescence spectra of salicylanilide 1a in $\mathrm{CH}_{3} \mathrm{CN}$ in the presence of increasing concentration of $\mathrm{H}_{2} \mathrm{PO}_{4}^{-}$(Figure S4).

5. Absorption and fluorescence spectra of salicylanilide $\mathbf{1 b}$ in $\mathrm{CH}_{3} \mathrm{CN}$ in the presence of increasing concentration of $\mathrm{H}_{2} \mathrm{PO}_{4}^{-}$(Figure S5). .54

6. Absorption and fluorescence spectra of salicylanilide $\mathbf{1 c}$ in $\mathrm{CH}_{3} \mathrm{CN}$ in the presence of increasing concentration of $\mathrm{H}_{2} \mathrm{PO}_{4}^{-}$(Figure S6).

7. Absorption and fluorescence spectra of salicylanilide $\mathbf{1 e}$ in $\mathrm{CH}_{3} \mathrm{CN}$ in the presence of increasing concentration of $\mathrm{H}_{2} \mathrm{PO}_{4}^{-}$(Figure S7). .S5

8. Absorption and fluorescence spectra of salicylanilide $\mathbf{1 f}$ in $\mathrm{CH}_{3} \mathrm{CN}$ in the presence of increasing

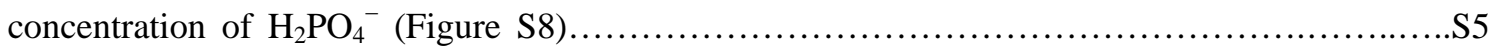

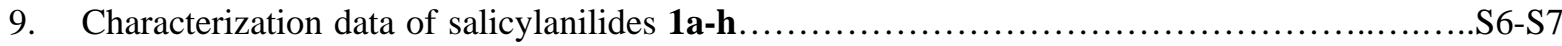

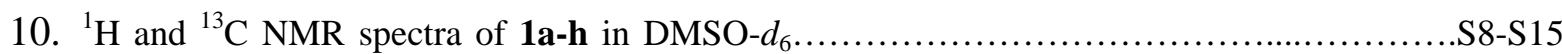




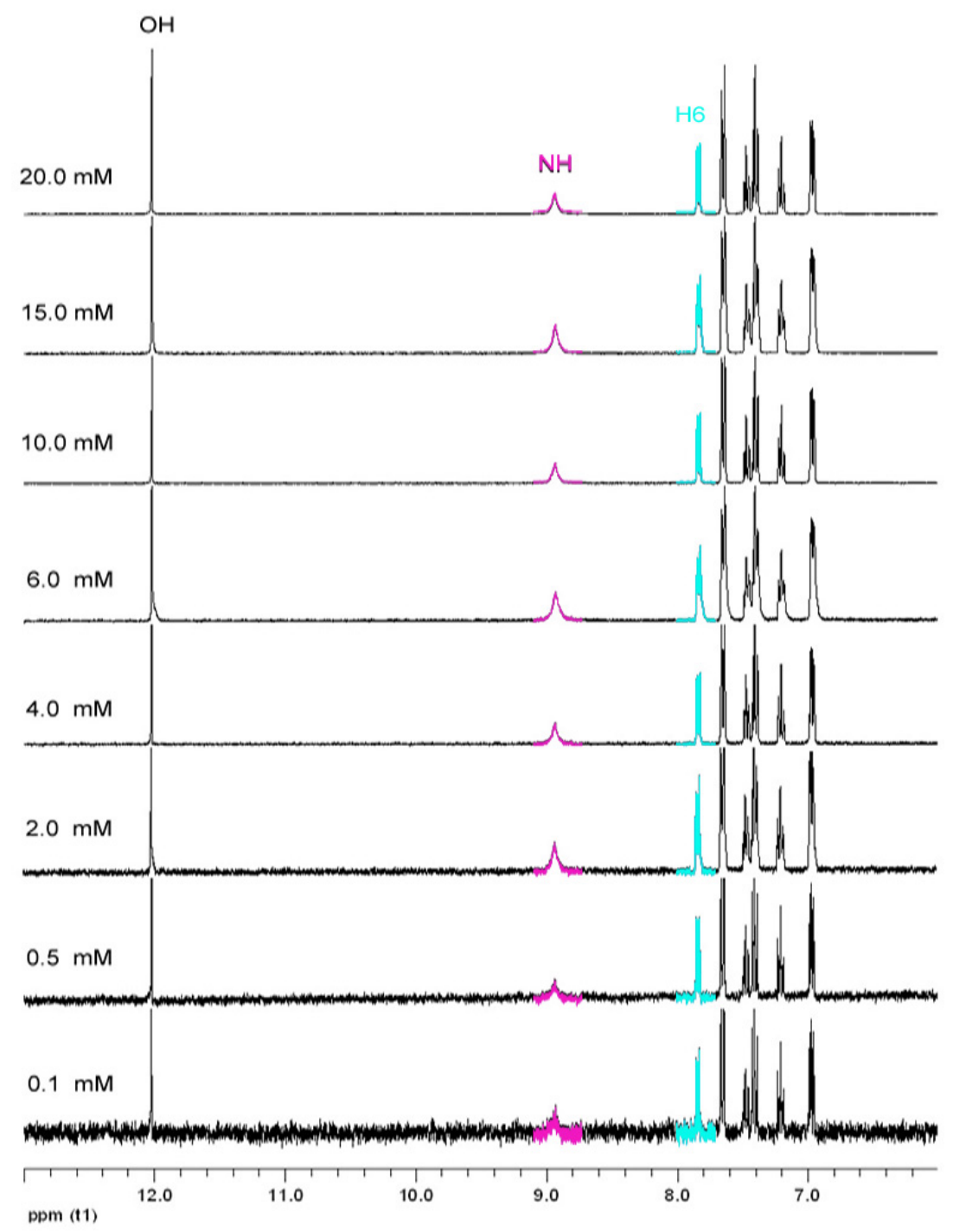

Figure S1. ${ }^{1} \mathrm{H}$ NMR spectra.of $\mathbf{1 d}$ in $\mathrm{CD}_{3} \mathrm{CN}$ at varied concentration. From bottom to top, concentration of $\mathbf{1 d}$ varies in order of $0.1,0.5,2.0,4.0,6.0,10.0,15.0$ to $20.0 \mathrm{mmol} \mathrm{L}^{-1}$.

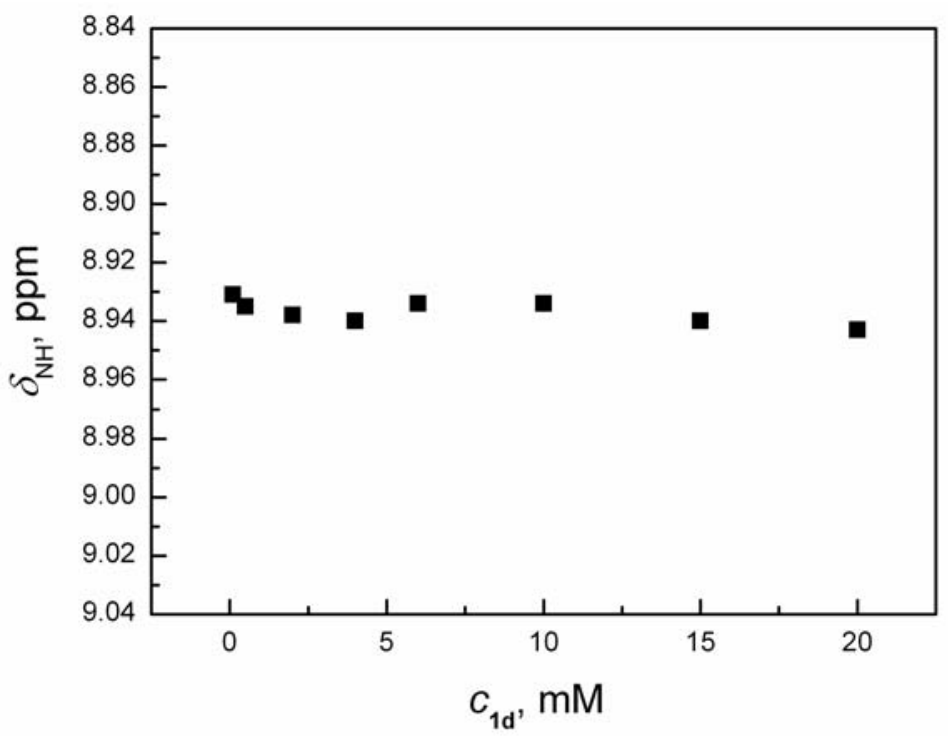

Figure S2. Chemical shift of amido NH proton of $\mathbf{1 d}$ as a function of $\mathbf{1 d}$ concentration in $\mathrm{CD}_{3} \mathrm{CN}$ 


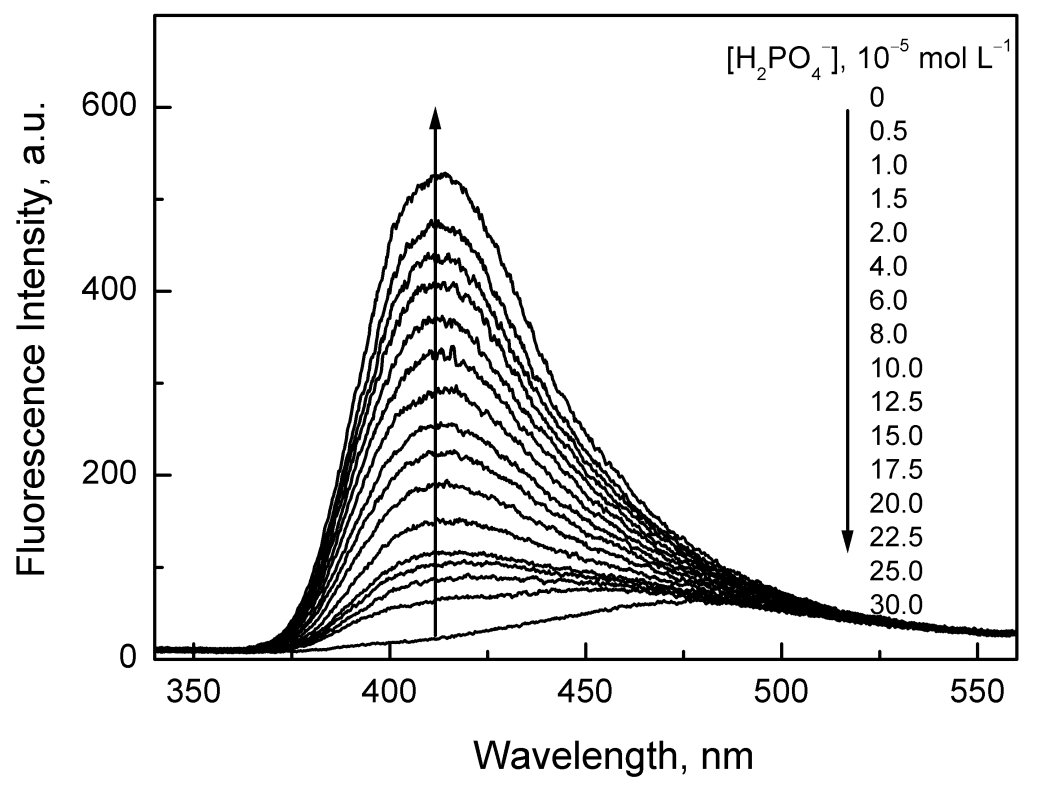

Figure S3. Fluorescence spectra of salicylanilide 1d in the presence of triethylamine in $\mathrm{CH}_{3} \mathrm{CN}$. [1d] $=1.0 \times 10^{-5} \mathrm{~mol} \mathrm{~L}^{-1}$. Excitation wavelength employed to record spectra was $290 \mathrm{~nm}$.

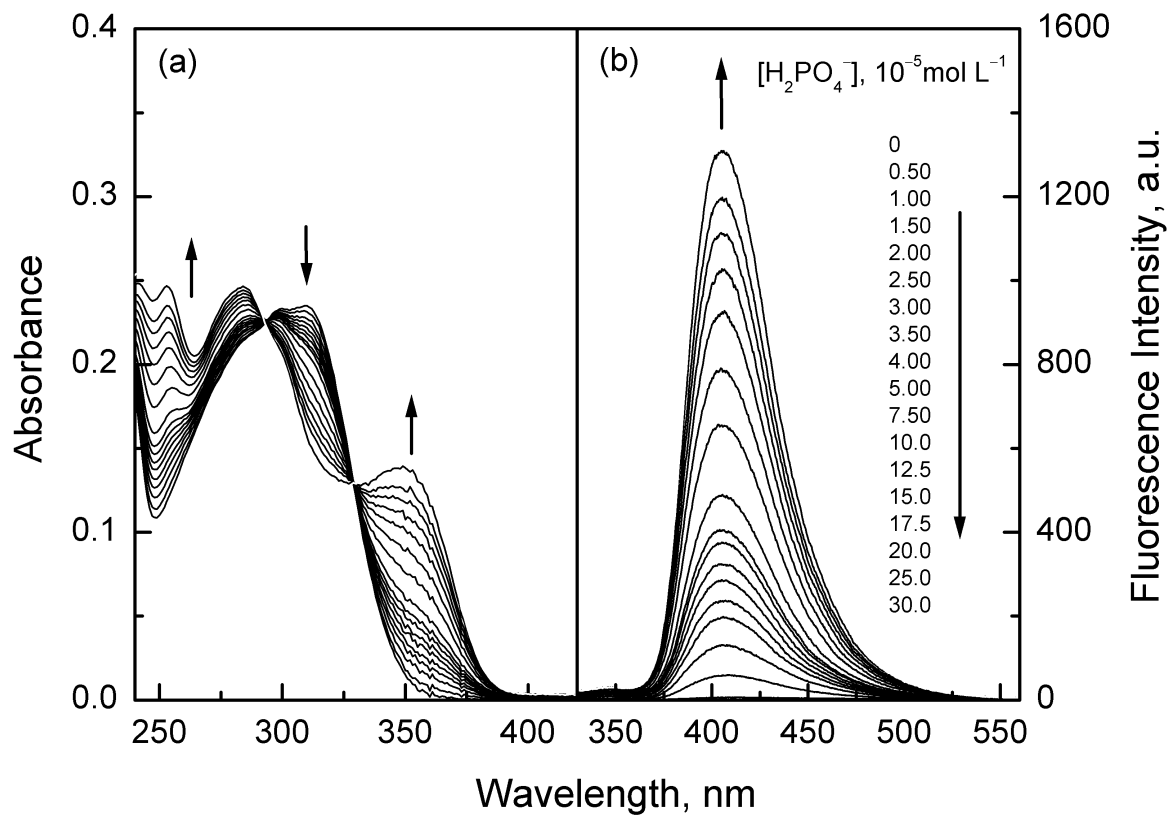

Figure S4. Absorption (a) and fluorescence (b) spectra of salicylanilide 1a in $\mathrm{CH}_{3} \mathrm{CN}$ in the presence of increasing concentration of $\mathrm{H}_{2} \mathrm{PO}_{4}^{-} . \quad[\mathbf{1 a}]=2.0 \times 10^{-5}$ (a) and $1.0 \times 10^{-5}$ (b) mol L $\mathrm{L}^{-1}$. Excitation wavelength employed to record spectra in (b) was $293 \mathrm{~nm}$, an isosbestic wavelength seen in (a). 


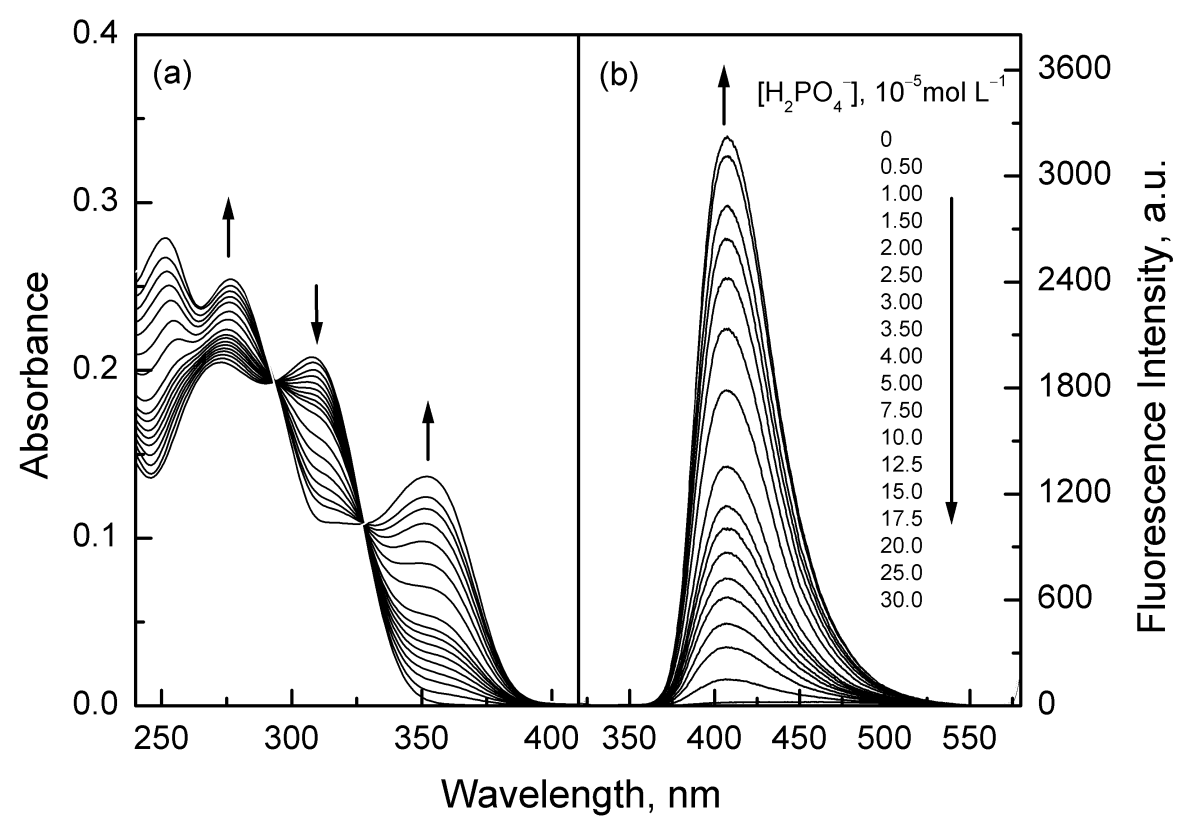

Figure S5. Absorption (a) and fluorescence (b) spectra of salicylanilide $\mathbf{1 b}$ in $\mathrm{CH}_{3} \mathrm{CN}$ in the presence of increasing concentration of $\mathrm{H}_{2} \mathrm{PO}_{4}{ }^{-} . \quad[\mathbf{1 b}]=2.0 \times 10^{-5}$ (a) and $1.0 \times 10^{-5}$ (b) mol L $\mathrm{L}^{-1}$. Excitation wavelength employed to record spectra in (b) was $293 \mathrm{~nm}$, an isosbestic wavelength seen in (a).

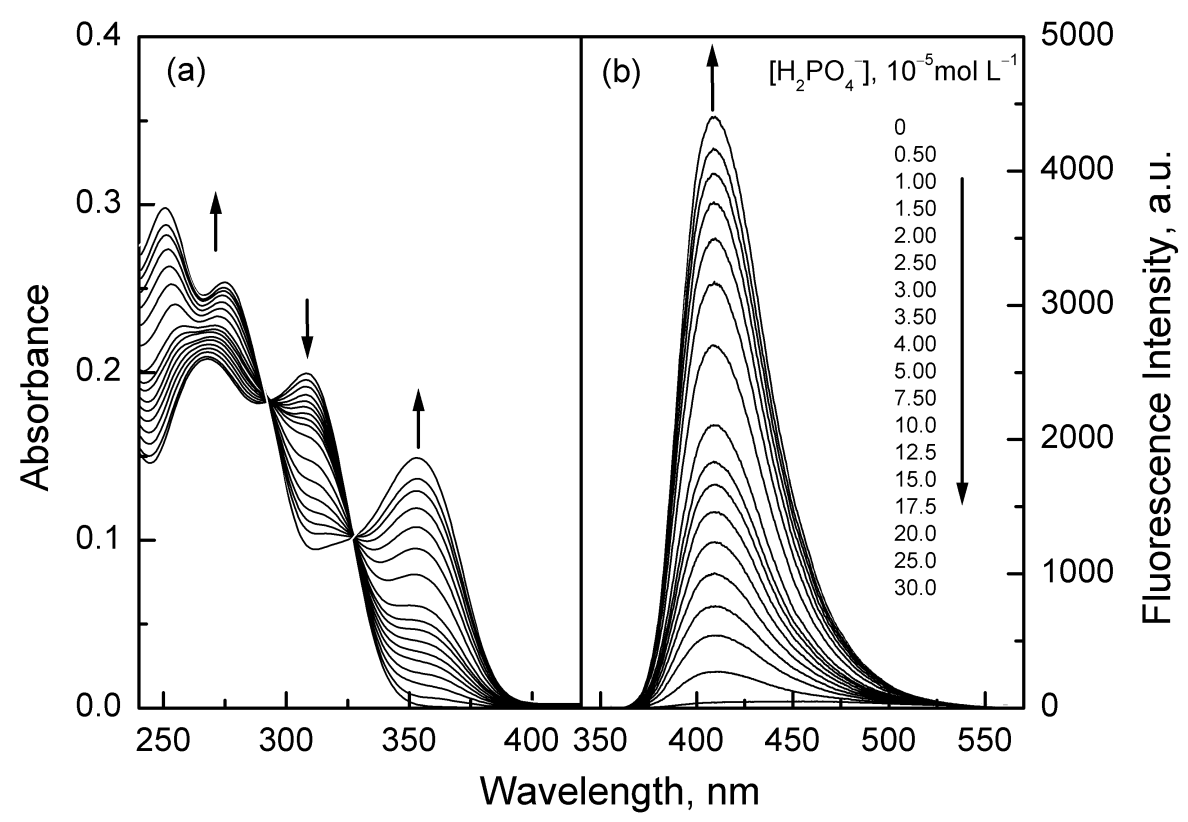

Figure S6. Absorption (a) and fluorescence (b) spectra of salicylanilide 1c in $\mathrm{CH}_{3} \mathrm{CN}$ in the presence of increasing concentration of $\mathrm{H}_{2} \mathrm{PO}_{4}^{-} . \quad[\mathbf{1 c}]=2.0 \times 10^{-5}$ (a) and $1.0 \times 10^{-5}$ (b) mol L $\mathrm{L}^{-1}$. Excitation wavelength employed to record spectra in (b) was $292 \mathrm{~nm}$, an isosbestic wavelength seen in (a). 


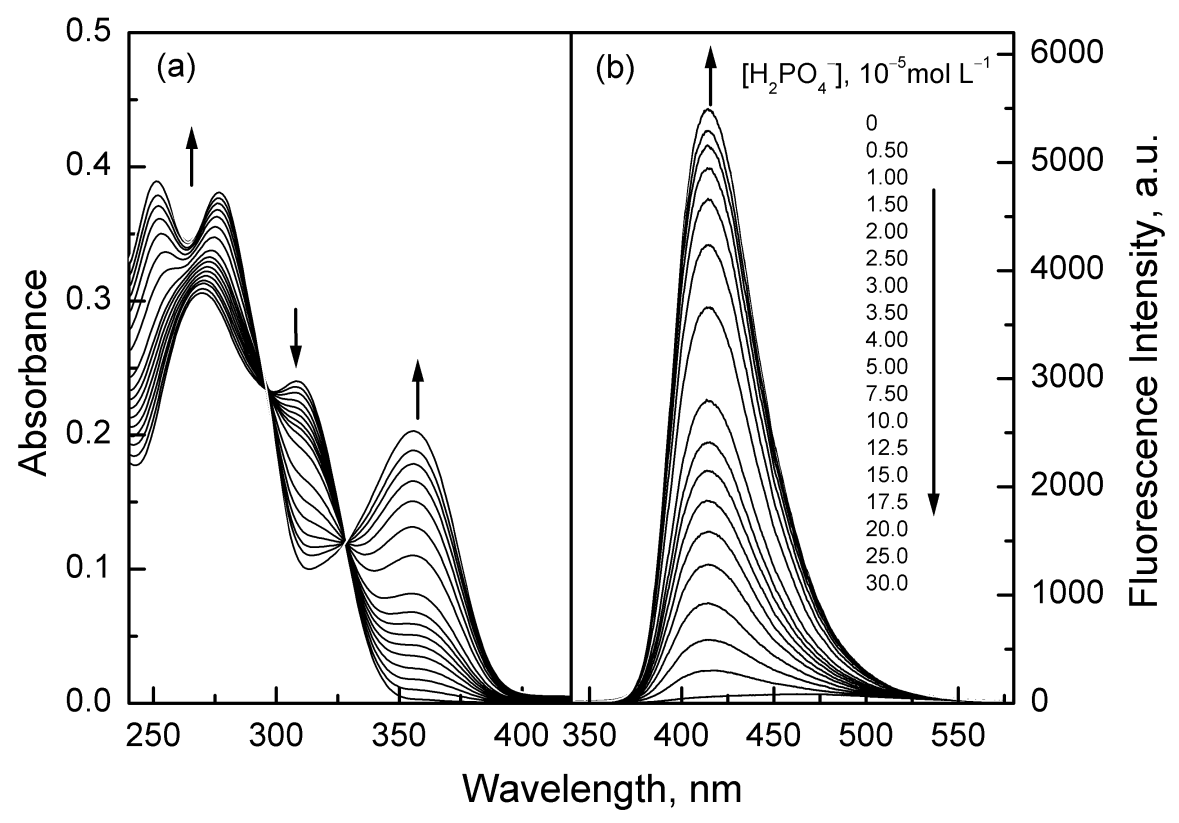

Figure S7. Absorption (a) and fluorescence (b) spectra of salicylanilide 1e in $\mathrm{CH}_{3} \mathrm{CN}$ in the presence of increasing concentration of $\mathrm{H}_{2} \mathrm{PO}_{4}{ }^{-} . \quad[\mathbf{1 e}]=2.0 \times 10^{-5}$ (a) and $1.0 \times 10^{-5}$ (b) mol L ${ }^{-1}$. Excitation wavelength employed to record spectra in (b) was $296 \mathrm{~nm}$, an isosbestic wavelength seen in (a).

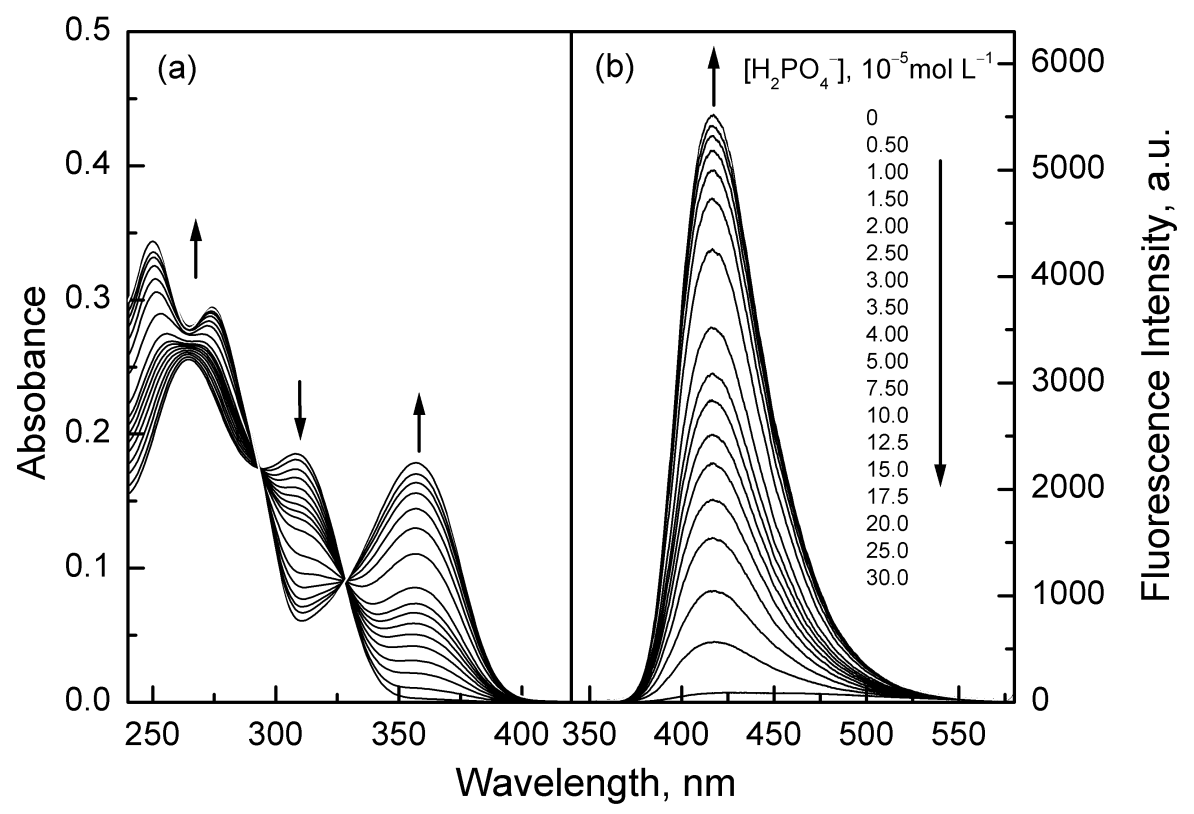

Figure S8. Absorption (a) and fluorescence (b) spectra of salicylanilide 1f in $\mathrm{CH}_{3} \mathrm{CN}$ in the presence of increasing concentration of $\mathrm{H}_{2} \mathrm{PO}_{4}{ }^{-}$. [ [1f $]=2.0 \times 10^{-5}$ (a) and $1.0 \times 10^{-5}$ (b) mol L $\mathrm{L}^{-1}$. Excitation wavelength employed to record spectra in (b) was $294 \mathrm{~nm}$, an isosbestic wavelength seen in (a). 


\section{Characterization data of salicylanilides 1a-h}

\section{2-Hydroxy- $N$-(4-methoxyphenyl)benzamide (1a)}

${ }^{1}$ H NMR (500MHz, DMSO- $d_{6}$ ): $\delta$ (ppm) 3.76 (s, 3H), 6.94-6.98 (m, 4H), 7.44 (t, 1H, $J=$ 7.5Hz), 7.59 (d, 2H, $J=8.0 \mathrm{~Hz}$ ), 7.97 (d, 1H, $J=7.5 \mathrm{~Hz}$ ), 10.29 (s, 1H), 12.01 (s, 1H).

${ }^{13}$ C NMR (125MHz, DMSO-d $\left.d_{6}\right): \delta$ (ppm) 166.7, 159.0, 156.1, 133.7, 131.0, 128.7, 122.9, 118.9, 117.3, 116.9, 113.9, 55.2 .

HRMS (ESI): calcd for $\left[\mathrm{C}_{14} \mathrm{H}_{14} \mathrm{NO}_{3}^{+}\right]$: 244.0973, found: 244.0978 .

\section{2-Hydroxy- $N$-(p-tolyl)benzamide (1b)}

${ }^{1}$ H NMR (500MHz, DMSO-d $)$ ): $\delta$ (ppm) 2.29 (s, 3H), 6.97-6.98 (m, 2H), 7.17 (d, 2H, $J=$ $7.0 \mathrm{~Hz}$ ), 7.44 (t, $1 \mathrm{H}, J=7.0 \mathrm{~Hz}), 7.58$ (d, 2H, $J=7.0 \mathrm{~Hz}), 7.97$ (d, $1 \mathrm{H}, J=7.0 \mathrm{~Hz}), 10.32$ (s, 1H), 11.90 (s, 1H).

${ }^{13}$ C NMR (125MHz, DMSO- $\left.d_{6}\right): \delta$ (ppm) 166.6, 158.7, 135.5, 133.7, 133.4, 129.2, 128.9, 121.1, 119.0, 117.3, 117, 2, 20.5.

HRMS (ESI): calcd for $\left[\mathrm{C}_{14} \mathrm{H}_{14} \mathrm{NO}_{2}^{+}\right]$: 228.1024, found: 228.1030 .

\section{2-Hydroxy- $N$-(m-tolyl)benzamide (1c)}

${ }^{1}$ H NMR (500MHz, DMSO- $d_{6}$ ): $\delta$ (ppm) 2.32 (s, 3H), 6.97-6.99 (m, 3H), 7.25 (t, 1H, $J=$ 7.5Hz), 7.44 (t, $1 \mathrm{H}, J=7.5 \mathrm{~Hz}), 7.49$ (d, 1H, $J=7.5 \mathrm{~Hz}$ ), 7.54 (s, 1H), 7.97 (d, 1H, $J=8.0 \mathrm{~Hz}$ ), 10.32 (s, 1H), 11.84 (s, 1H).

${ }^{13}$ C NMR (125MHz, DMSO-d ${ }_{6}$ ): $\delta$ (ppm) 166.6, 158.6, 138.1, 138.0, 133.7, 129.0, 128.6, 124.9, 121.5, 119.0, 118.2, 117.4, 117.3, 21.1.

HRMS (ESI): calcd for $\left[\mathrm{C}_{14} \mathrm{H}_{14} \mathrm{NO}_{2}{ }^{+}\right]$: 228.1024, found: 228.1029.

\section{2-Hydroxy- $N$-phenylbenzamide (1d)}

${ }^{1}$ H NMR (500MHz, DMSO- $d_{6}$ ): $\delta$ (ppm) 6.96-7.00 (m, 2H), 7.15 (t, $\left.1 \mathrm{H}, J=7.5 \mathrm{~Hz}\right), 7.38$ (t, $2 \mathrm{H}, J=8.5 \mathrm{~Hz}), 7.44$ (t, $1 \mathrm{H}, J=8.0 \mathrm{~Hz}), 7.70$ (d, $2 \mathrm{H}, J=8.5 \mathrm{~Hz}), 7.96(\mathrm{~d}, 1 \mathrm{H}, J=8.0 \mathrm{~Hz})$, 10.38 (s, 1H), 11.80 (s, 1H).

${ }^{13}$ C NMR (125MHz, DMSO-d $\left.d_{6}\right): \delta$ (ppm) 166.6, 158.5, 138.1, 133.7, 129.1, 128.8, 124.2, 121.0, 119.1, 117.5, 117.2.

HRMS (ESI): calcd for $\left[\mathrm{C}_{13} \mathrm{H}_{12} \mathrm{NO}_{2}{ }^{+}\right]$: 214.0868, found: 214.0868 .

\section{$\mathrm{N}$-(4-Chlorophenyl)-2-hydroxybenzamide (1e)}

${ }^{1}$ H NMR (500MHz, DMSO- $d_{6}$ ): $\delta$ (ppm) 6.96-6.70 (m, 2H), 7.42-7.44 (m, 3H), 7.75 (d, 2H, $J=8.0 \mathrm{~Hz}$ ), 7.92 (d, $1 \mathrm{H}, J=7.5 \mathrm{~Hz}$ ), 10.45 (s, 1H), 11.65 (s, 1H).

${ }^{13}$ C NMR (125MHz, DMSO- $\left.d_{6}\right): \delta$ (ppm) 166.5, 158.2, 137.2, 133.7, 129.1, 128.6, 127.7, 122.4, 119.1, 117.2. 
HRMS (ESI): calcd for $\left[\mathrm{C}_{13} \mathrm{H}_{11} \mathrm{ClNO}_{2}{ }^{+}\right]$: 248.0478, found: 248.0486 .

$N$-(3-Chlorophenyl)-2-hydroxybenzamide (1f)

${ }^{1}$ H NMR (500MHz, DMSO-d $\left.d_{6}\right): \delta(\mathrm{ppm})$ 6.97-7.00 (m, 2H), 7.19 (d, $\left.1 \mathrm{H}, J=7.0 \mathrm{~Hz}\right)$, 7.38-7.44 (m, 2H), 7.62 (d, 1H, $J=7.5 \mathrm{~Hz}$ ), 7.90-7.94 (m, 2H), 10.47 (s, 1H), 11.55 (s, 1H).

${ }^{13}$ C NMR (125MHz, DMSO-d $\left.d_{6}\right): \delta$ (ppm) 166.6, 158.1, 139.8, 133.7, 133.1, 130.4, 129.2, 123.7, 120.2, 119.1, 117.8, 117.2 .

HRMS (ESI): calcd for $\left[\mathrm{C}_{13} \mathrm{H}_{11} \mathrm{ClNO}_{2}{ }^{+}\right]$: 248.0478, found: 248.0482 .

4-(2-Hydroxybenzoylamino)benzoic acid methyl ester (1g)

${ }^{1}$ H NMR (500MHz, DMSO- $d_{6}$ ): $\delta$ (ppm) 3.801 (s, 3H), 6.92-6.97 (m, 2H), 7.40 (t, 1H, $J=$ 7.5Hz), 7.84-7.88 (m, 3H), 7.92 (d, 2H, $J=8.5 \mathrm{~Hz}), 10.58$ (s, 1H), 11.47 (s, 1H).

${ }^{13}$ C NMR (125MHz, DMSO- $\left.d_{6}\right): \delta$ (ppm) 166.4, 165.8, 157.7, 142.8, 133.7, 130.2, 129.5, 124.6, 119.9, 119.2, 118.3, 117.1, 51.9.

HRMS (ESI): calcd for $\left[\mathrm{C}_{15} \mathrm{H}_{14} \mathrm{NO}_{4}{ }^{+}\right]$: 272.0923, found: 272.0927.

$N$-(4-Cyanophenyl)-2-hydroxybenzamide (1h)

${ }^{1}$ H NMR (400MHz, DMSO-d $\left.d_{6}\right): \delta(\mathrm{ppm})$ 6.96-7.02 (m, 2H), $7.45(\mathrm{t}, 1 \mathrm{H}, J=7.2 \mathrm{~Hz})$, 7.82-7.88 (m, 3H), 7.93 (d, 2H, $J=8.4 \mathrm{~Hz}), 10.67$ (s, 1H), 11.39 (s, 1H).

${ }^{13}$ C NMR (100MHz, DMSO- $\left.d_{6}\right): \delta$ (ppm) 166.5, 157.5, 142.7, 133.7, 133.2, 129.5, 120.4, 119.2, 119.0, 118.6, 117.1, 105.6.

HRMS (ESI): calcd for $\left[\mathrm{C}_{14} \mathrm{H}_{11} \mathrm{~N}_{2} \mathrm{O}_{2}^{+}\right]$: 239.0820, found: 239.0821 . 
2-Hydroxy- $N$-(4-methoxyphenyl)benzamide (1a)

${ }^{1} \mathrm{H}$ NMR (500MHz, DMSO- $d_{6}$ )
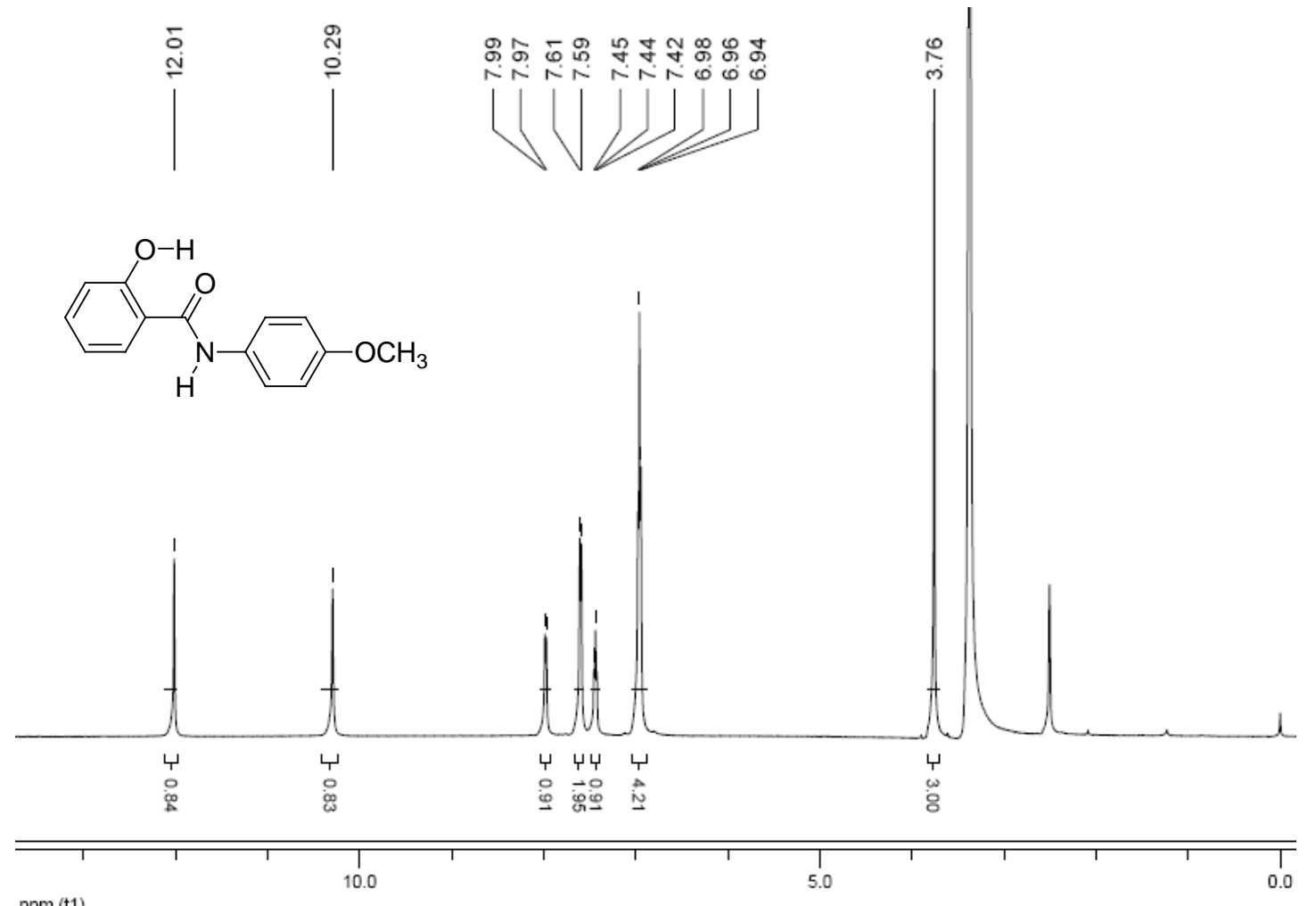

ppm (t1)

${ }^{13} \mathrm{C}$ NMR (125MHz, DMSO- $d_{6}$ )

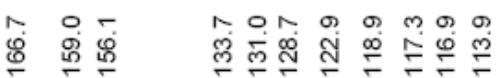
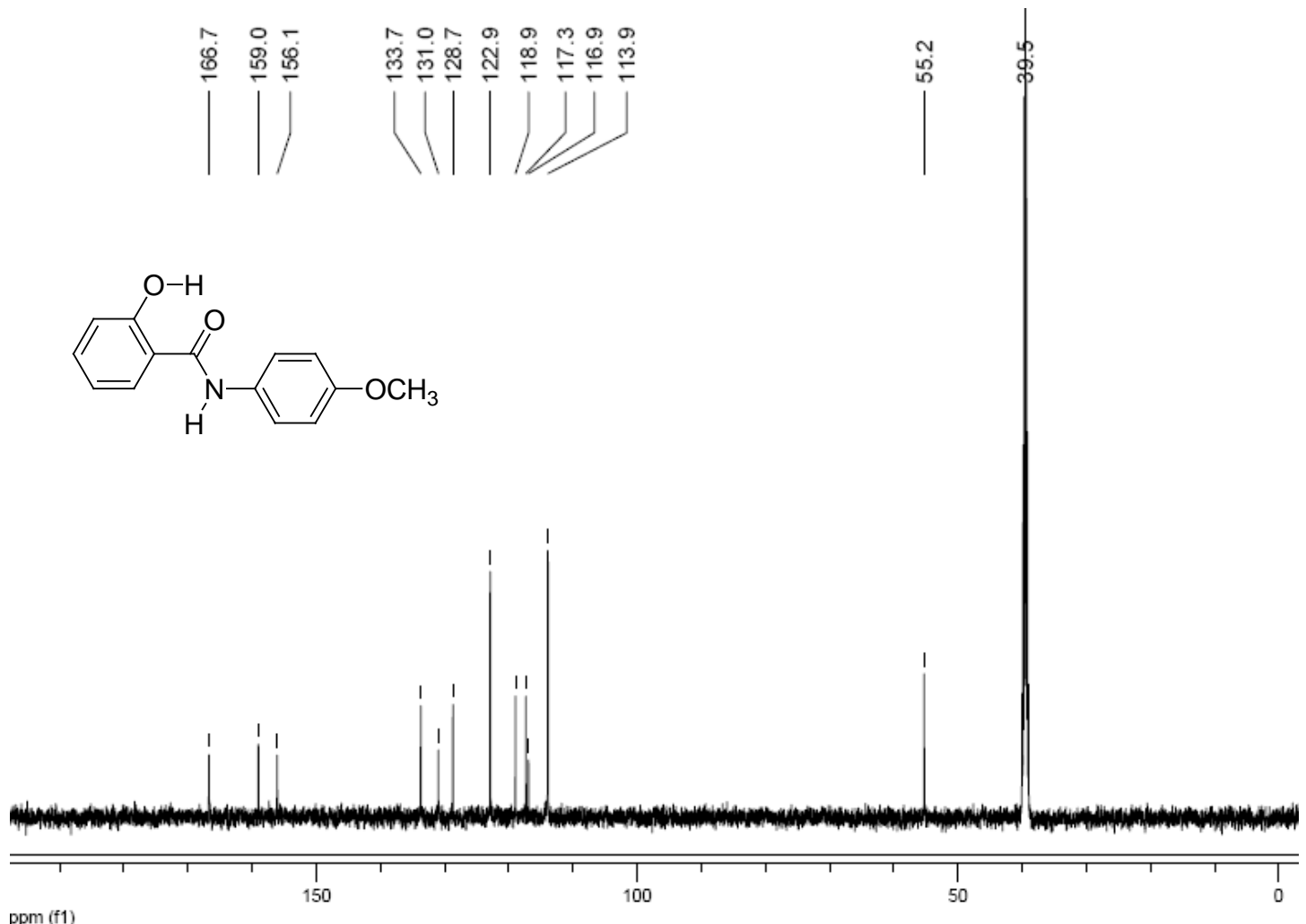


\section{2-Hydroxy- $N$-(p-tolyl)benzamide (1b)}

${ }^{1} \mathrm{H}$ NMR $\left(500 \mathrm{MHz}\right.$, DMSO- $d_{6}$ )

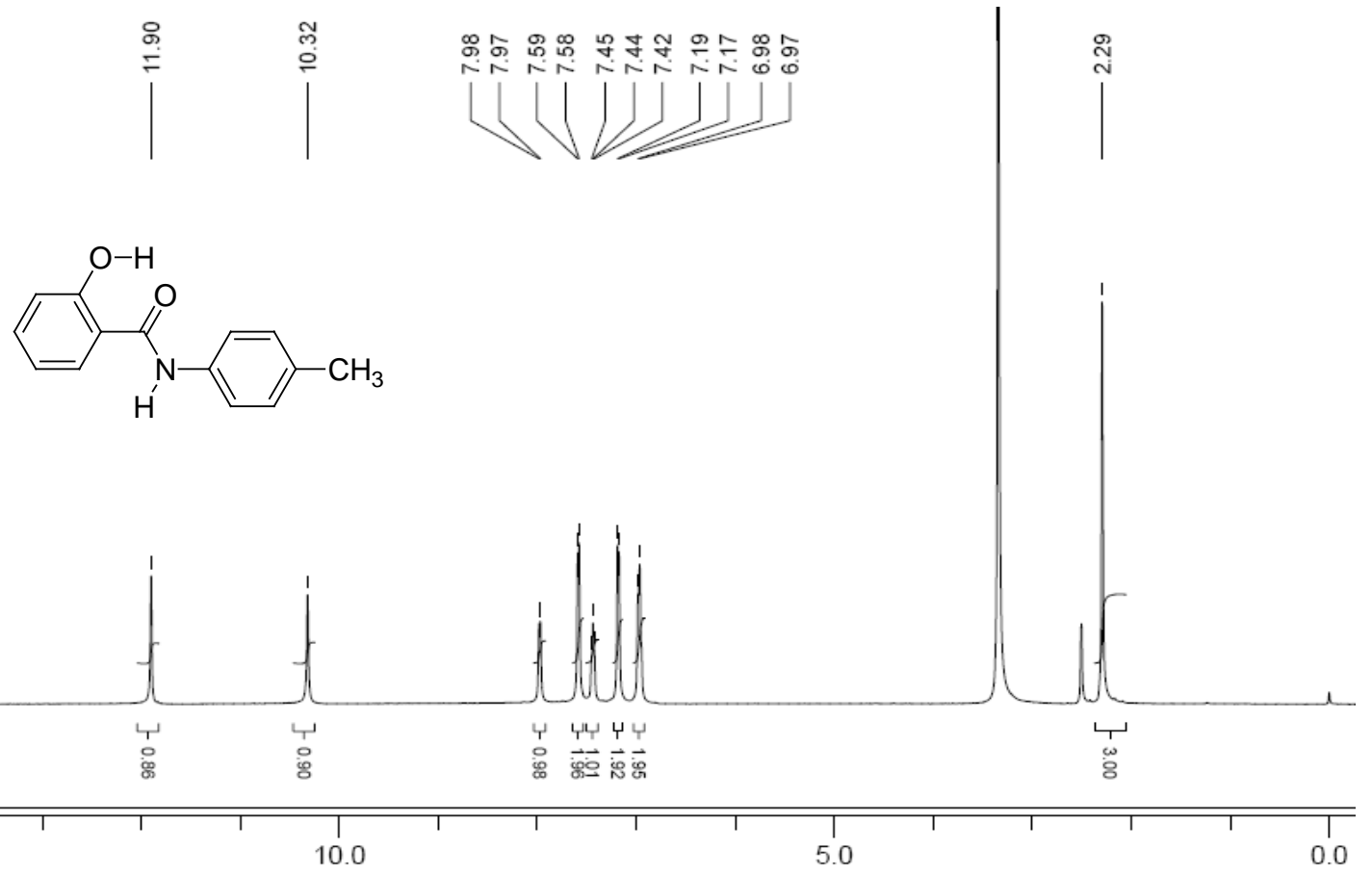

ppm (f1)

${ }^{13} \mathrm{C}$ NMR $\left(125 \mathrm{MHz}\right.$, DMSO- $\left.d_{6}\right)$
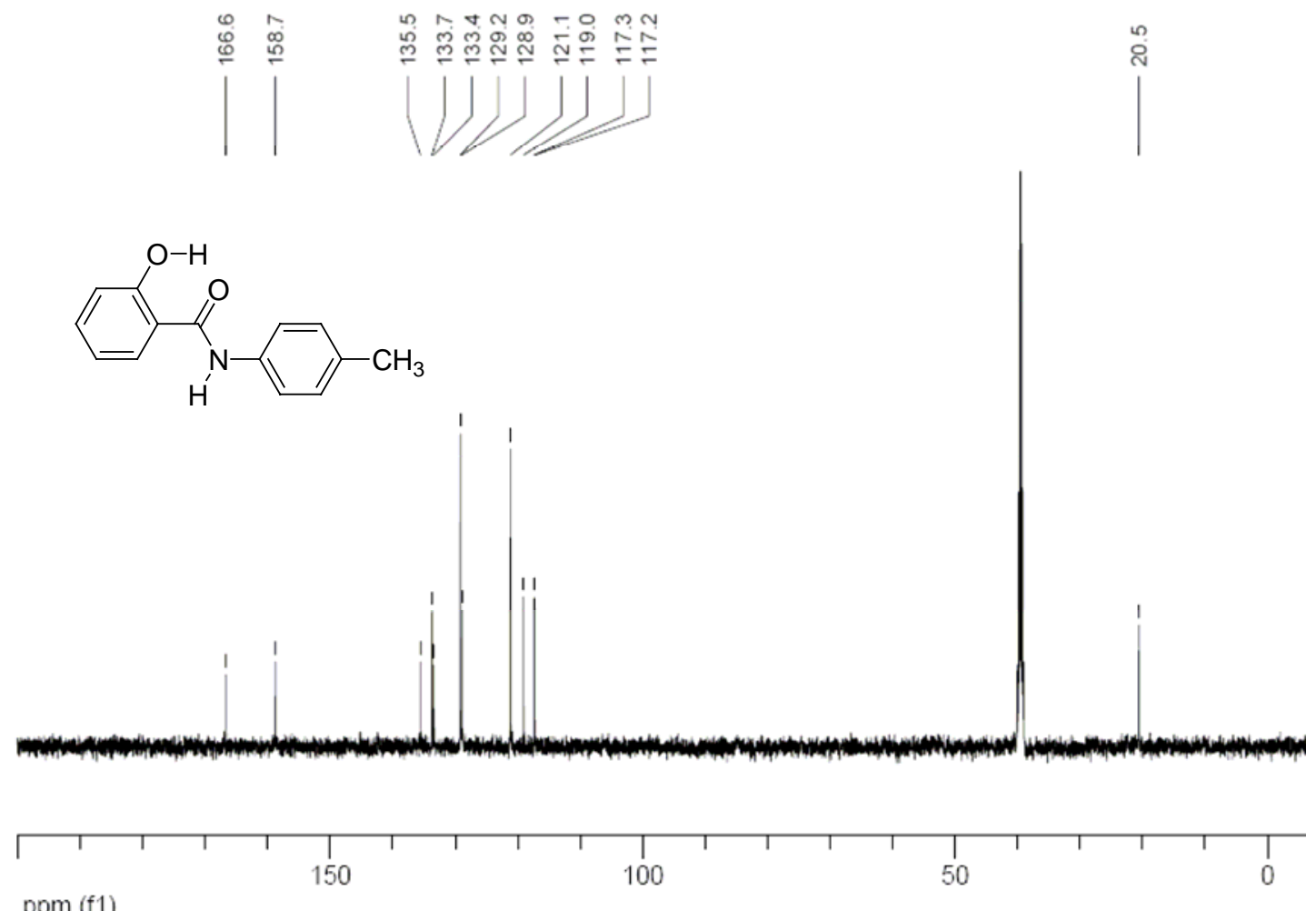

ppm (f1) 


\section{2-Hydroxy- $N$-(m-tolyl)benzamide (1c)}

${ }^{1} \mathrm{H}$ NMR (500MHz, DMSO- $d_{6}$ )
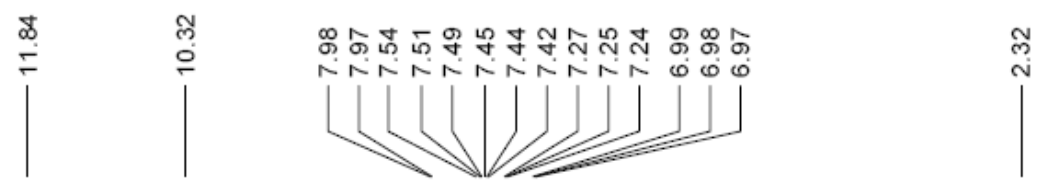<smiles>Cc1cccc(NC(=O)c2ccccc2O)c1</smiles>

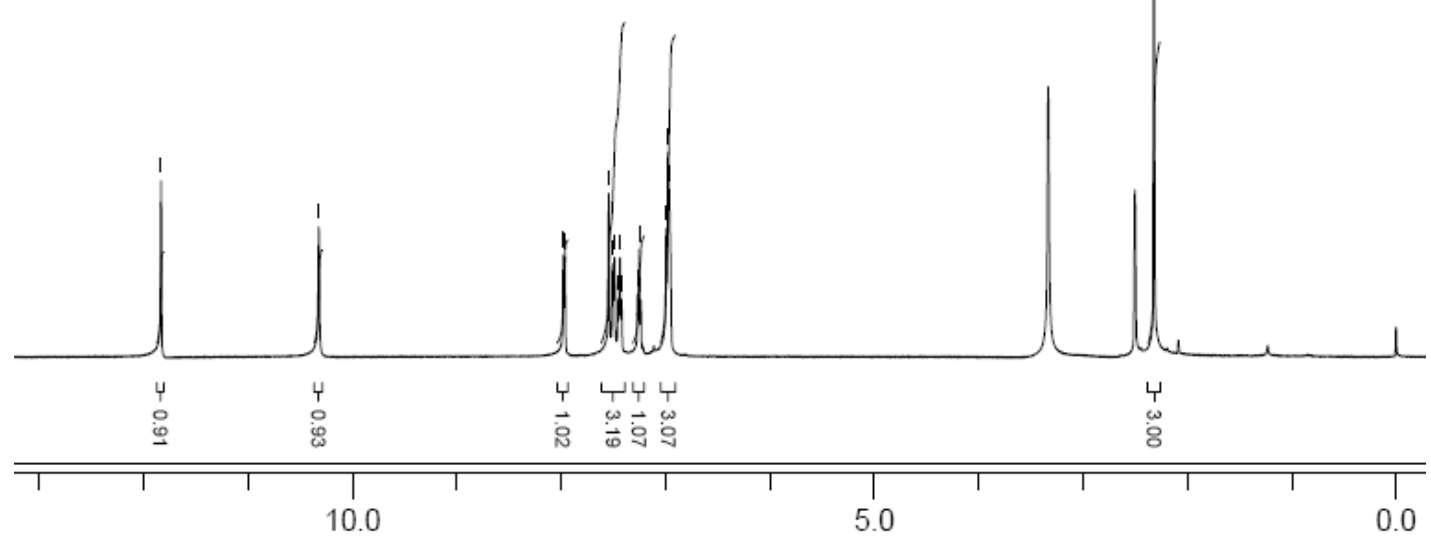

ppm (t1)

${ }^{13}$ C NMR (125MHz, DMSO- $d_{6}$ )
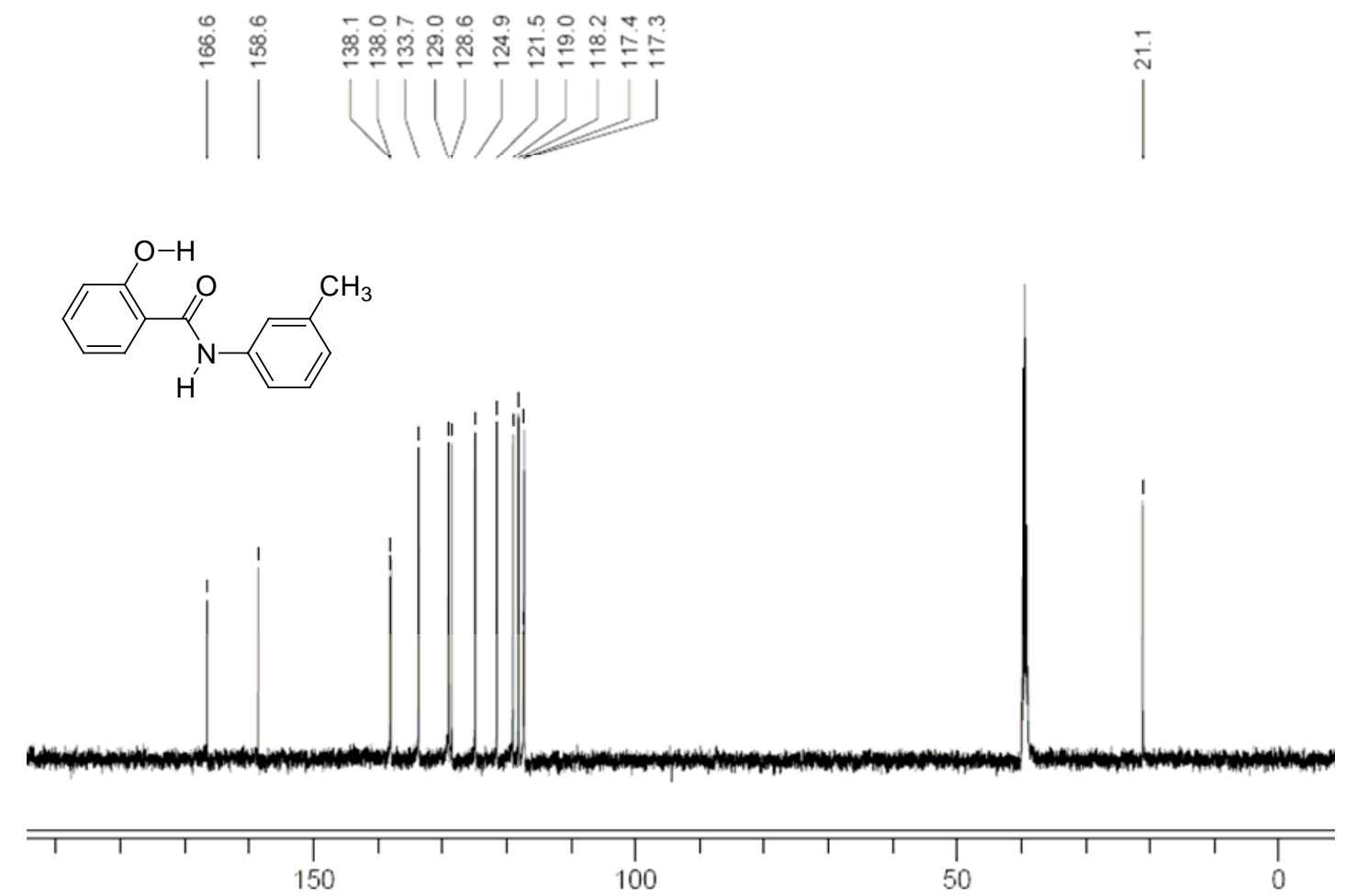

ppm (t1) 


\section{2-Hydroxy- $N$-phenylbenzamide (1d)}

${ }^{1} \mathrm{H}$ NMR (500MHz, DMSO- $d_{6}$ )
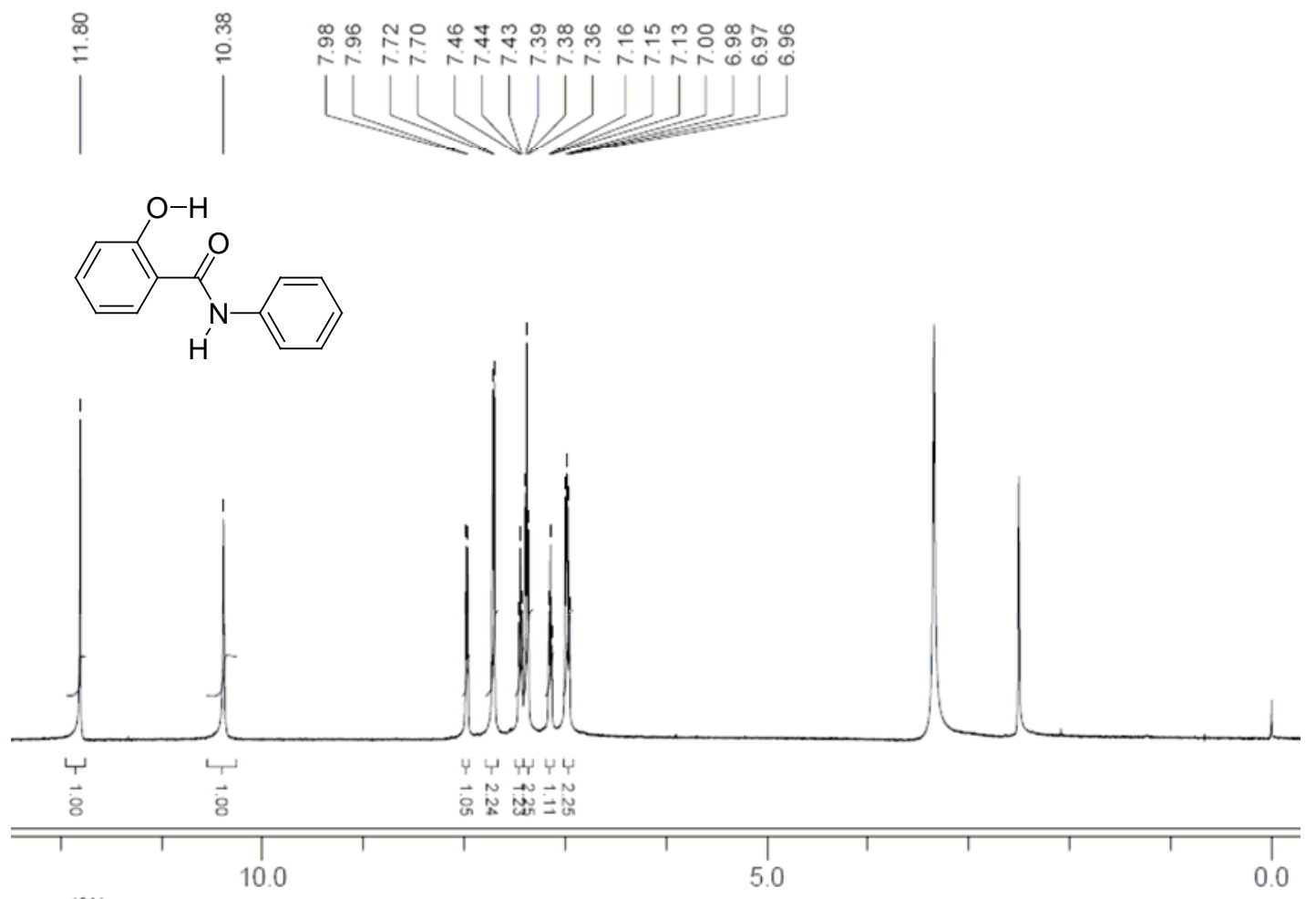

$\mathrm{ppm}(\mathrm{f} 1)$

${ }^{13} \mathrm{C}$ NMR (125MHz, DMSO- $d_{6}$ )

@<smiles>O=C(Nc1ccccc1)c1ccccc1O</smiles>

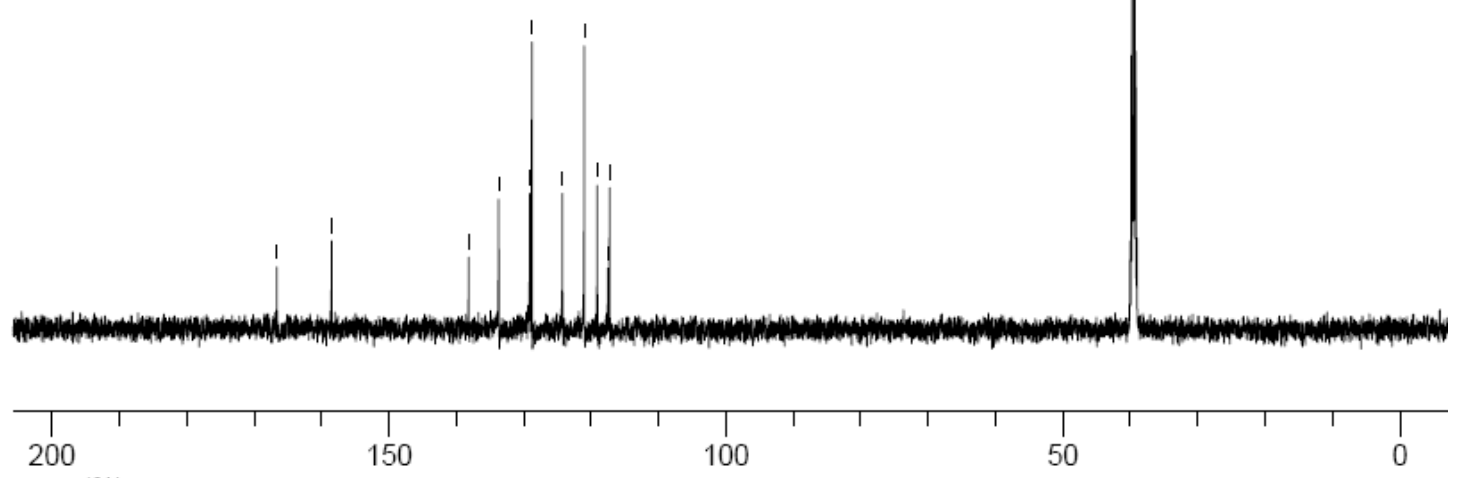

ppm (f1) 


\section{$N$-(4-Chlorophenyl)-2-hydroxybenzamide (1e)}

${ }^{1} \mathrm{H}$ NMR (500MHz, DMSO- $d_{6}$ )
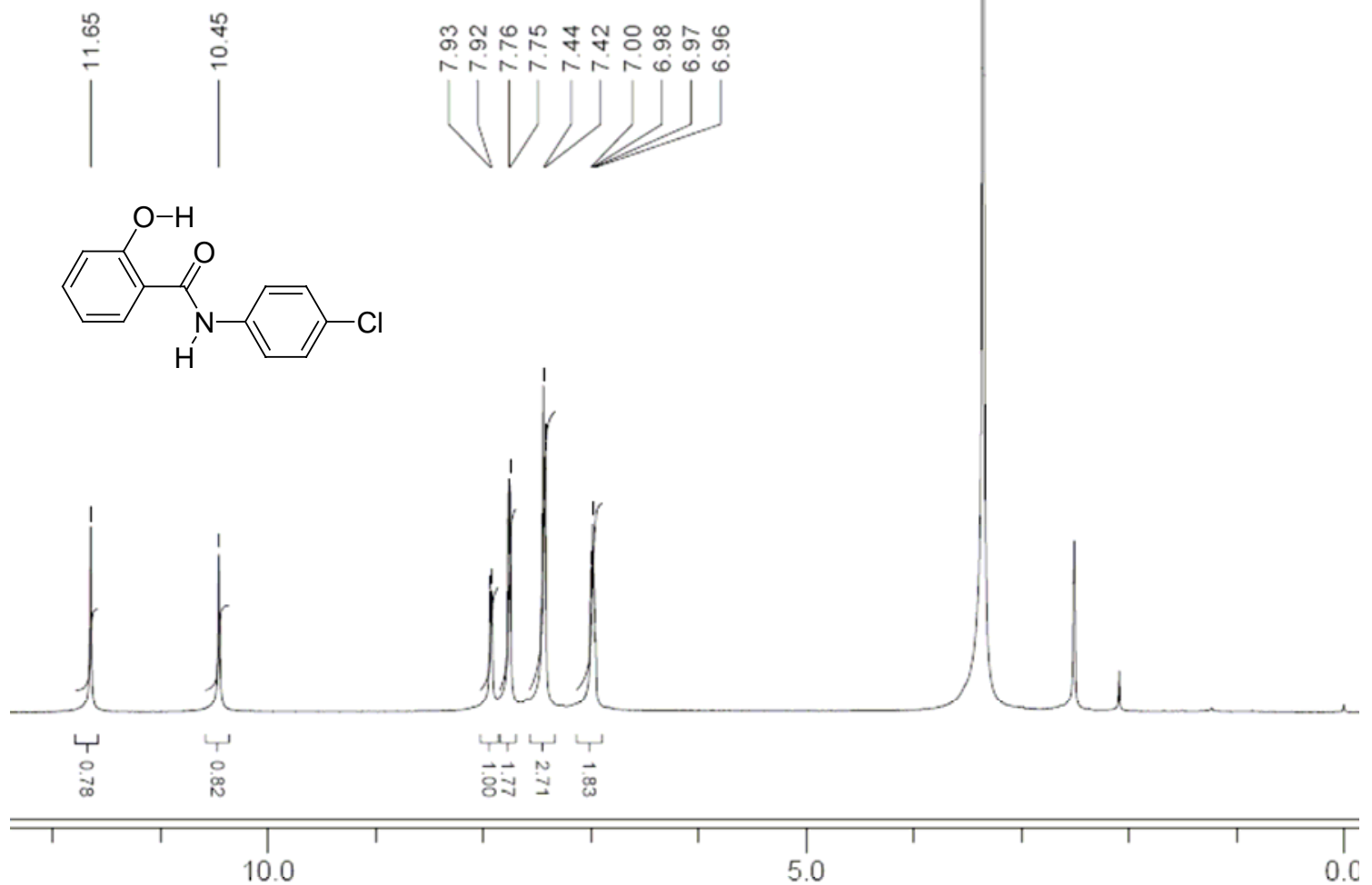

ppm (t1)

${ }^{13}$ C NMR (125MHz, DMSO- $d_{6}$ )
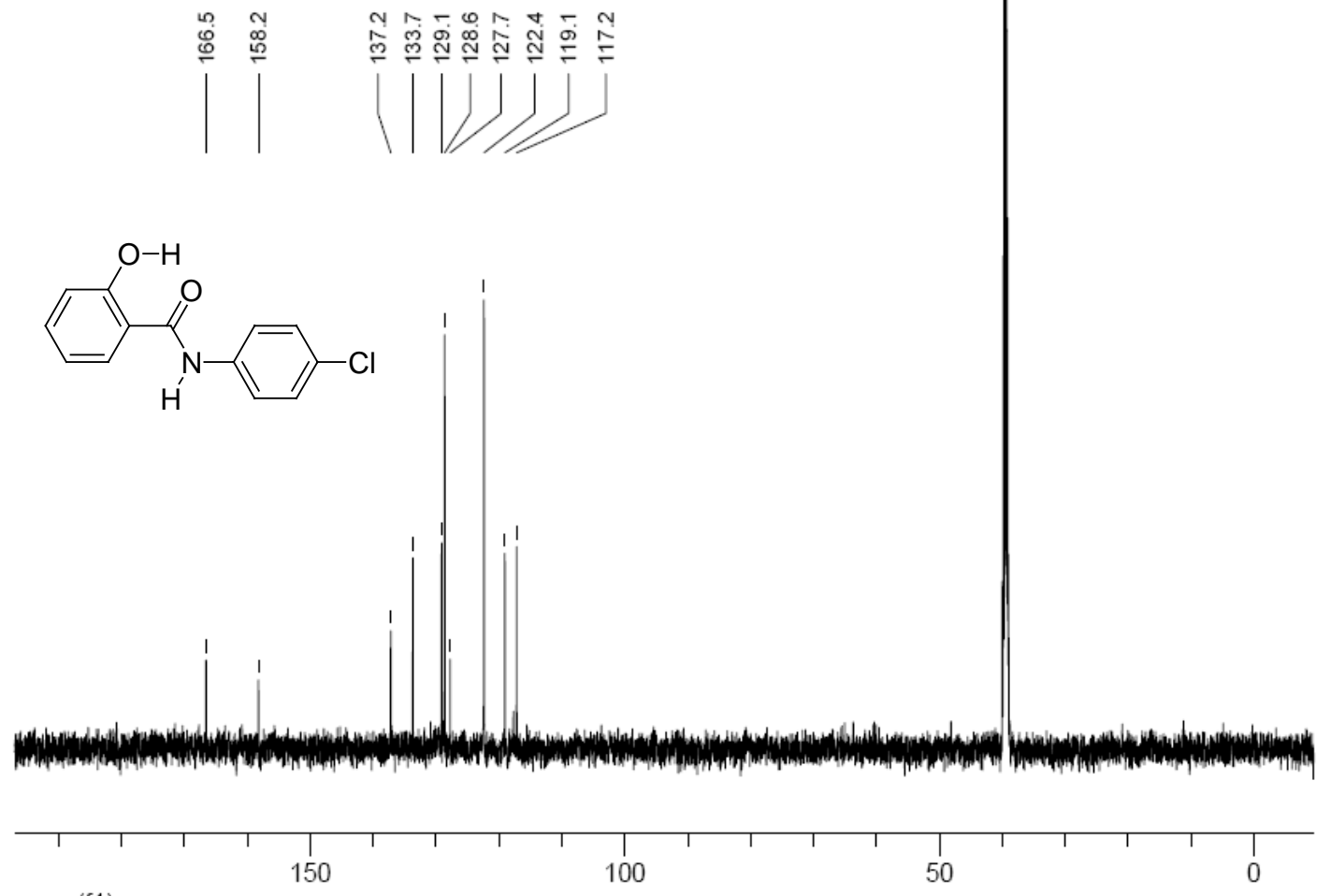

ppm (f1) 


\section{$N$-(3-Chlorophenyl)-2-hydroxybenzamide (1f)}

${ }^{1} \mathrm{H}$ NMR (500MHz, DMSO- $d_{6}$ )
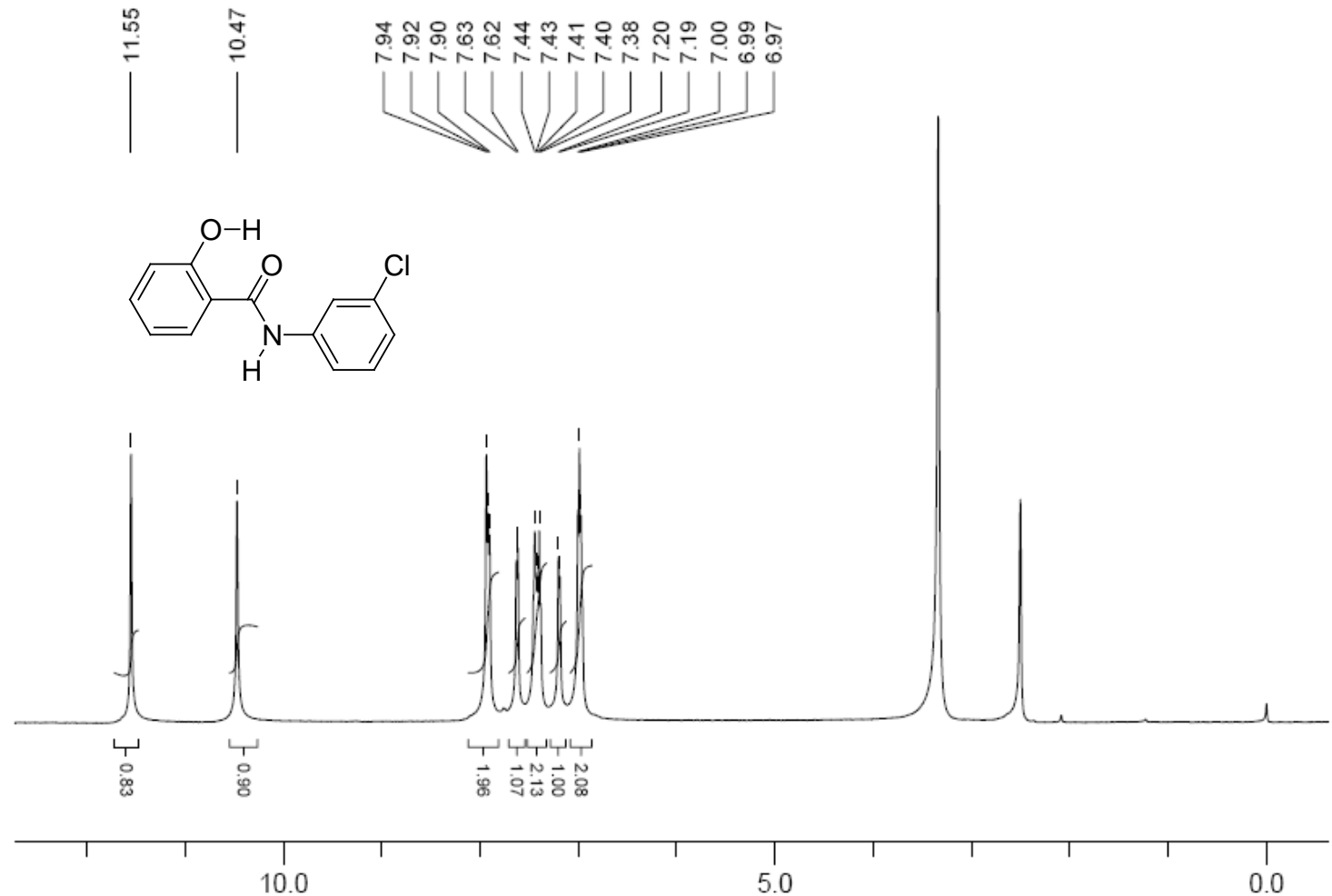

ppm (f1)

${ }^{13} \mathrm{C}$ NMR $\left(125 \mathrm{MHz}\right.$, DMSO- $\left.d_{6}\right)$

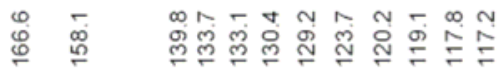
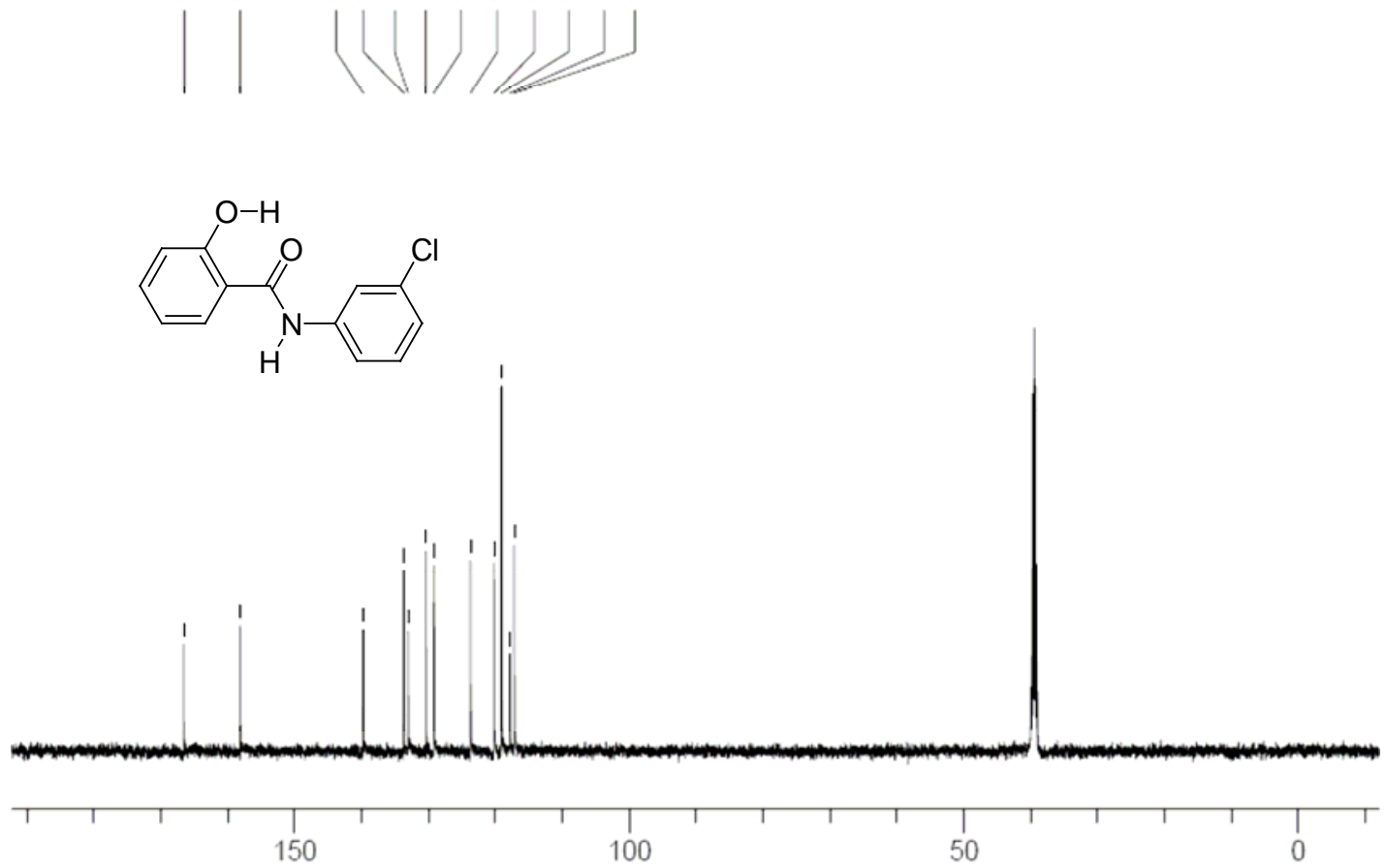

ppm (t1) 


\section{4-(2-Hydroxybenzoylamino)benzoic acid methyl ester (1g)}

${ }^{1} \mathrm{H}$ NMR (500MHz, DMSO- $d_{6}$ )

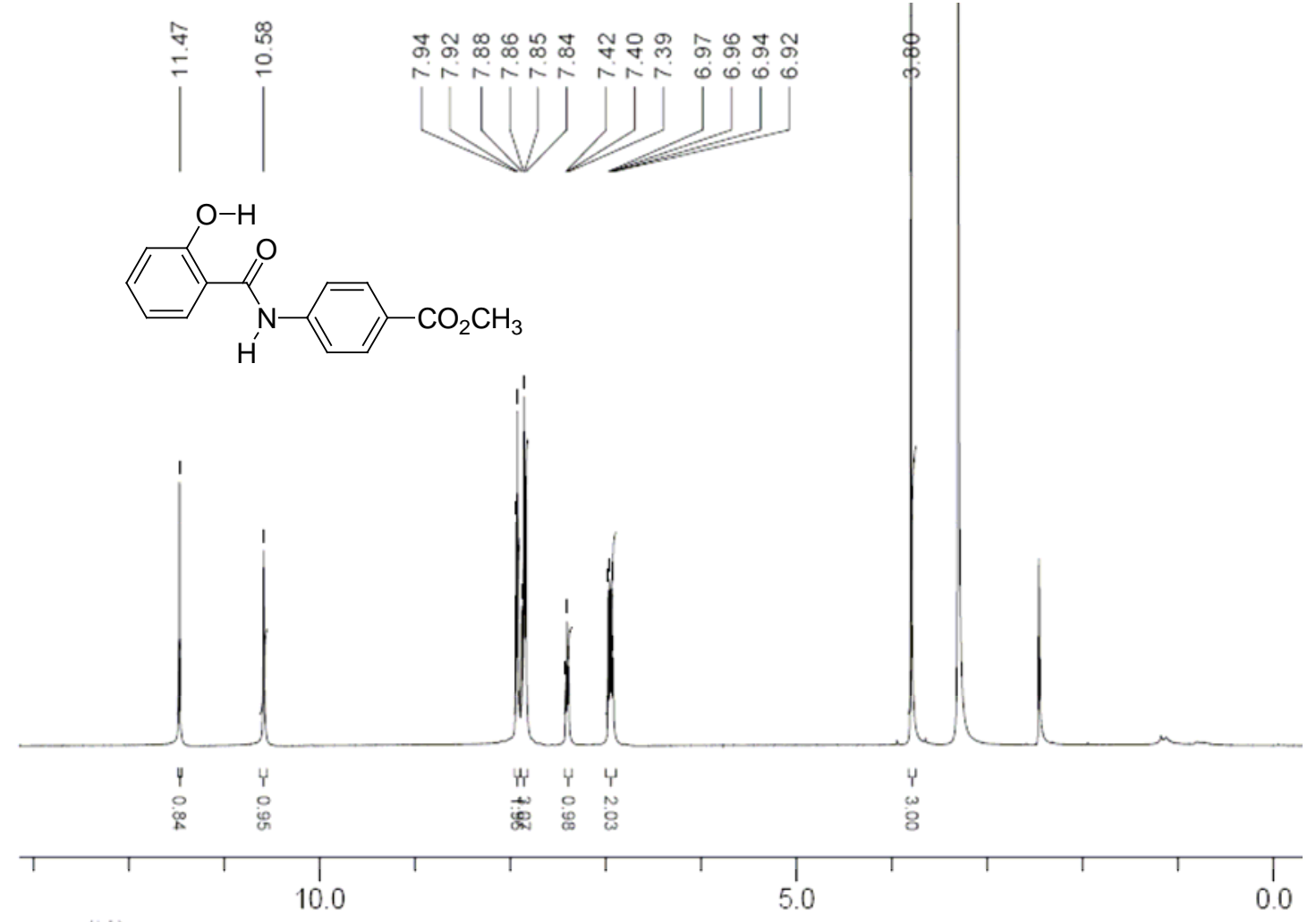

$\mathrm{ppm}(\mathrm{t} 1)$

${ }^{13}$ C NMR (125MHz, DMSO- $d_{6}$ )
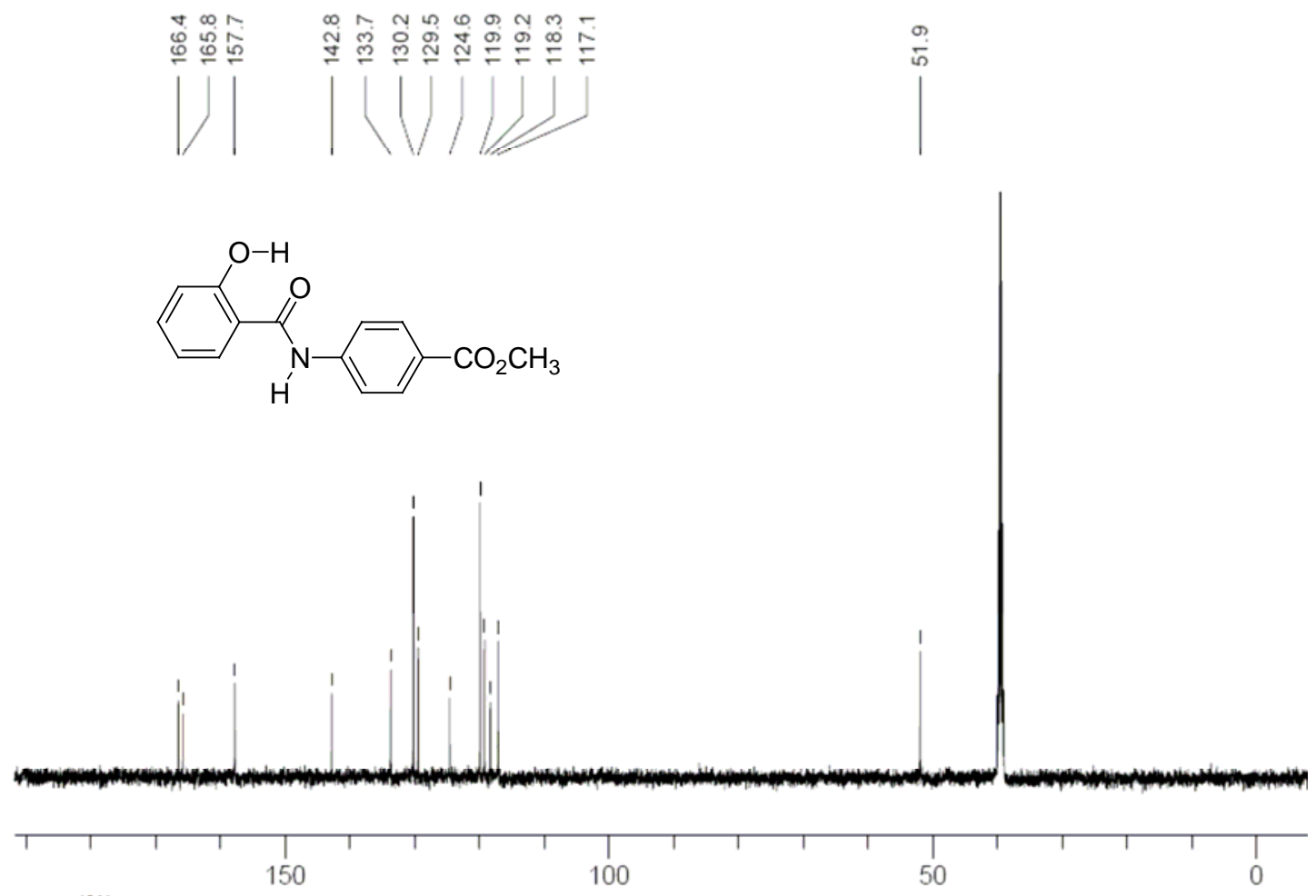

ppm (f1) 


\section{N-(4-Cyanophenyl)-2-hydroxybenzamide (1h)}

${ }^{1} \mathrm{H}$ NMR (400MHz, DMSO- $d_{6}$ )
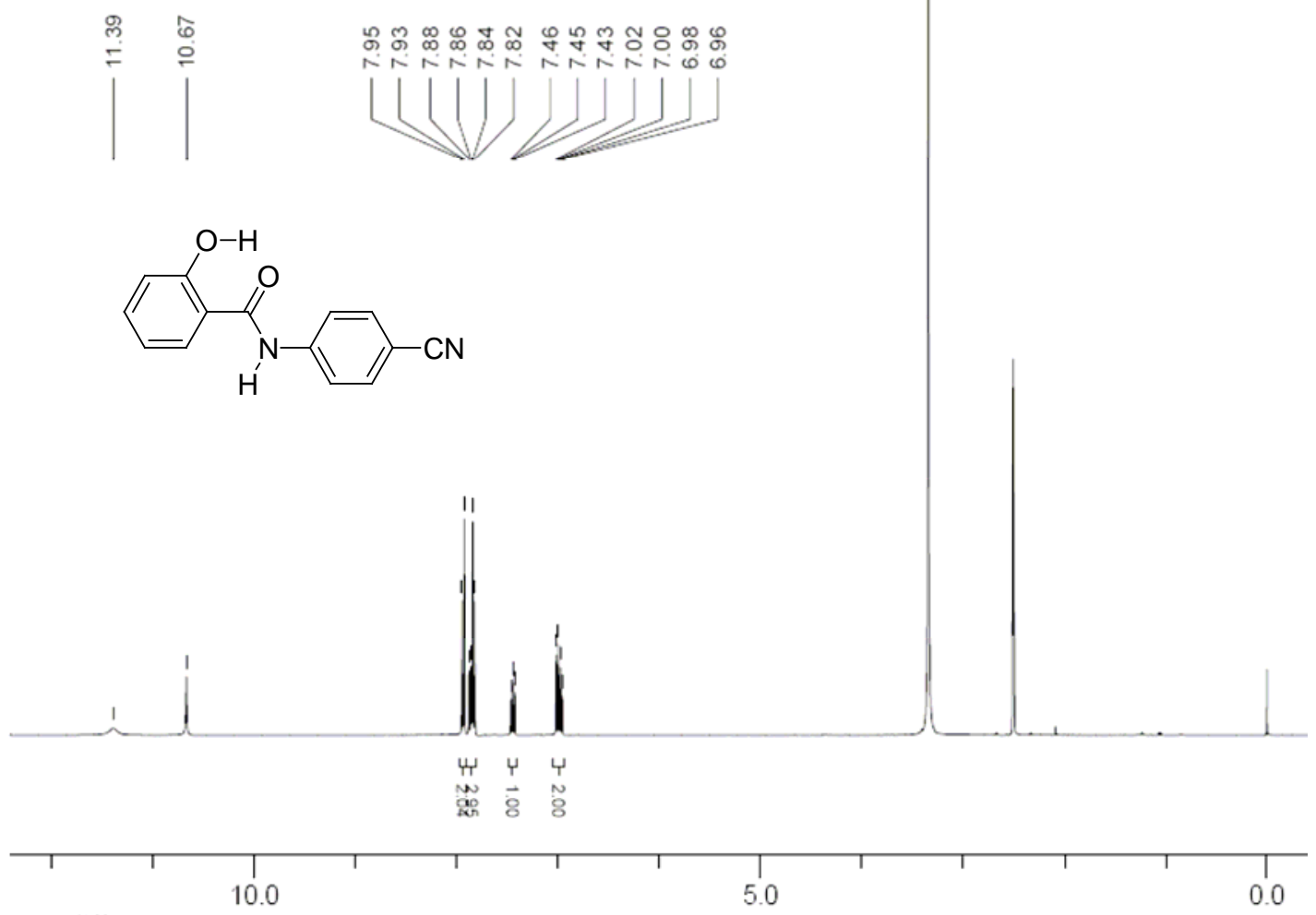

ppm (t1)

${ }^{13} \mathrm{C}$ NMR (100MHz, DMSO- $\left.d_{6}\right)$
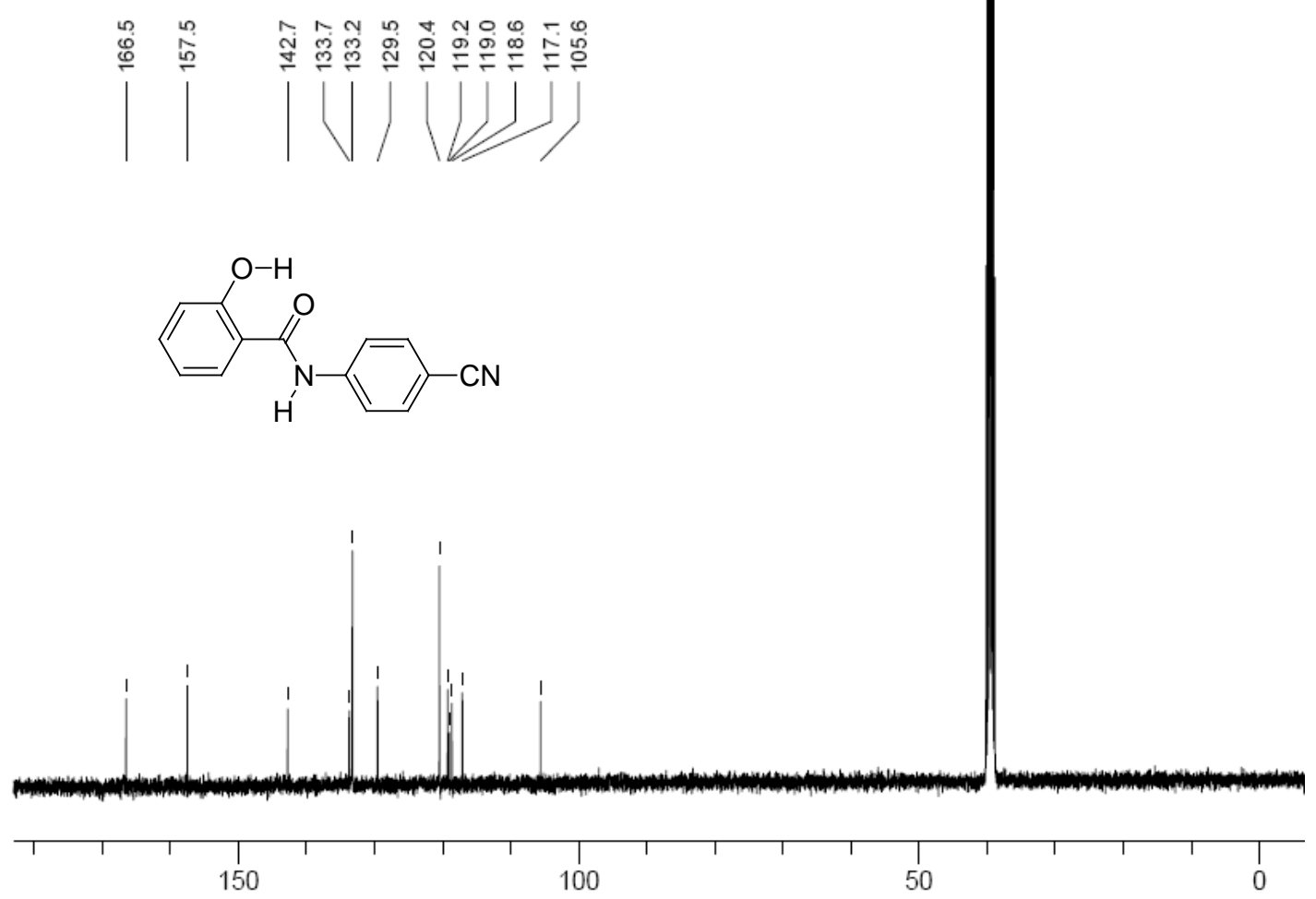

ppm (t1) 